\title{
A ballistic $p n$ junction in suspended graphene with split bottom gates
}

\author{
Anya L. Grushina, ${ }^{1}$ Dong-Keun $\mathrm{Ki}^{1}{ }^{1}$ and Alberto F. Morpurgo ${ }^{1,}$ a) \\ Départment de Physique de la Matiére Condensée (DPMC) and Group of Applied \\ Physics (GAP), University of Geneva, 24 Quai Ernest-Ansermet, CH1205 Genéve, \\ Switzerland
}

(Dated: 20 September 2018)

We have developed a process to fabricate suspended graphene devices with local bottom gates, and tested it by realizing electrostatically controlled $p n$ junctions on a suspended graphene mono-layer nearly $2 \mu \mathrm{m}$ long. Measurements as a function of gate voltage, magnetic field, bias, and temperature exhibit characteristic Fabry-Perot oscillations in the cavities formed by the $p n$ junction and each of the contacts, with transport occurring in the ballistic regime. Our results demonstrate the possibility to achieve a high degree of control on the local electronic properties of ultra-clean suspended graphene layers, a key aspect for the realization of new graphene nanostructures.

PACS numbers: 73.63.-b, 72.80.Vp, 73.40.-c, 07.60.Ly

a) Alberto.Morpurgo@unige.ch 
Technical developments in device fabrication are essential to perform transport experiments revealing the intrinsic electronic properties of graphene. Suspended graphene devices $^{1,2}$ and devices with hexagonal boron nitride $(\mathrm{hBN})$ as supporting substrate ${ }^{3,4}$ provide clear examples. The same is true for double-gated devices, in which graphene is not in direct contact with any dielectric material. ${ }^{5-7}$ Indeed, these devices have allowed the observation of phenomena such as the fractional quantum Hall effect, ${ }^{8-11}$ new interaction-induced symmetry broken states in bilayers, ${ }^{5,6}$ and manifestations of ballistic transport. ${ }^{12-14}$ Even more advanced experiments would be possible if double-gating on suspended devices could be performed locally. In bilayer graphene, for instance, local double gating would allow the study of topological confinement, ${ }^{15}$ and the realization of fully electrostatically tunable $p n$ junctions, of interest to generate or detect light at continuously tunable frequencies in the $\mathrm{THz}$ to mid infrared range. As an essential step towards the realization of these new structures, here we describe a technique to fabricate high-quality suspended graphene devices with local bottom gates and apply it to the realization of an electrostatically tunable, ballistic $p n$ junction in monolayer graphene.

The fabrication process is illustrated schematically in Fig. 1. The first step consists in preparing the bottom gates in the desired configuration -in the present case, a simple single strip- on a doped silicon substrate covered with $300 \mathrm{~nm} \mathrm{SiO}_{2}$, by using conventional techniques (electron-beam lithography, Ti/Au evaporation, and lift-off). Next, a 450-nmthick layer of polydimethylglutarimide (PMGI)-based lift-off resist (LOR, MicroChem) is spun onto the substrate (Fig. 1(a)). LOR resist is chosen because it is not only compatible with all subsequent micro-fabrication processes, but also it can be exposed with an electron beam and developed away to suspend graphene at the end of the fabrication process. ${ }^{16-18}$

As a second step, a graphene flake is transferred onto the LOR layer, and positioned on to the bottom gate (Fig. 1(b)). To this end, we adapted a technique developed to fabricate graphene/hBN heterostructures. ${ }^{3}$ Specifically, graphene is exfoliated using an adhesive tape and placed on a different substrate, previously coated with a layer of water soluble polymer (a 9 wt.\% poly(4-styrenesulfonic acid) solution in water) and a layer of PMMA. ${ }^{19}$ After the desired flake is identified under an optical microscope, the substrate is immersed in water, causing the water-soluble polymer to dissolve and the PMMA to float. The floating PMMA is retrieved using a plastic support, which is then mounted onto a micro-manipulator under an optical microscope. This enables the graphene flake to be transferred onto the LOR, 
a

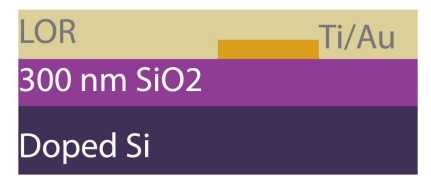

b

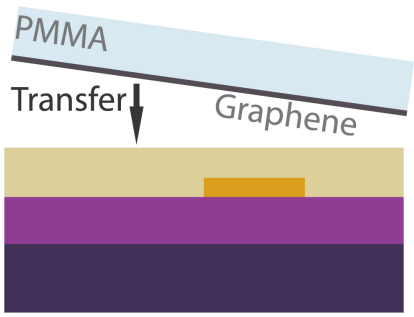

C

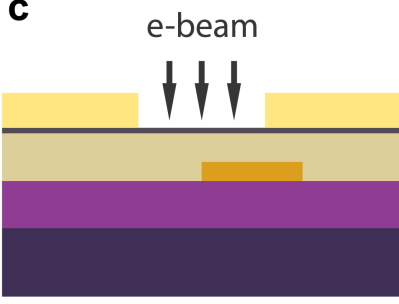

d

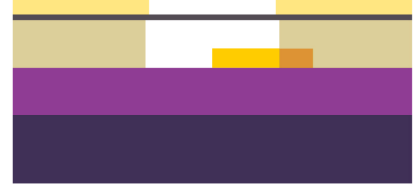

e

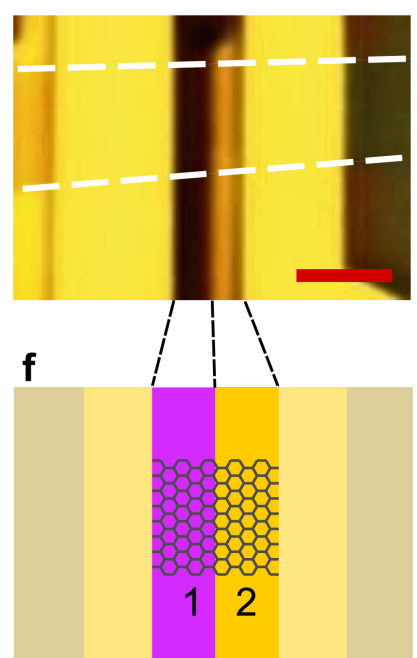

FIG. 1. (Color online) Schematic summary of the fabrication process (drawings not to scale). (a) A target substrate with predefined gate electrodes $(10 \mathrm{~nm} \mathrm{Ti} / 30 \mathrm{~nm} \mathrm{Au})$ is covered with a 450nm-thick LOR layer. (b) A graphene flake on a PMMA support is transferred onto LOR, aligned to the bottom electrode. (c-d) Graphene is contacted and the underlying LOR exposed with an electron beam, to achieve suspension. (e) Optical microscope image of the device (the dashed lines indicate the edges of the graphene flake); the local bottom gate is visible under the right electrode (the bar is $2 \mu \mathrm{m}$ long). (e) Schematic top view denoting the regions 1 and 2 (coupled primarily to the two different gate electrodes).

aligned to the bottom gate with a precision of a few microns (Fig. 1(b)). After securing it by heating at $105^{\circ} \mathrm{C}$ for 40 minutes, the flake is contacted with Ti/Au electrodes $(10 / 60 \mathrm{~nm}$ thick) defined by conventional electron-beam lithography, metal evaporation, and lift-off (for PMMA on LOR, development and lift-off are done using Xylene, ${ }^{16-18}$ at room temperature and $\sim 90{ }^{\circ} \mathrm{C}$, respectively). In the final step, the LOR under the graphene layer is exposed with an electron beam (Fig. 1(c)) and developed away to achieve the suspension (Fig. $1(\mathrm{~d})) \cdot{ }^{16-18}$

We have applied this technique to suspend a $1.8 \mu \mathrm{m}$ long graphene monolayer over a 

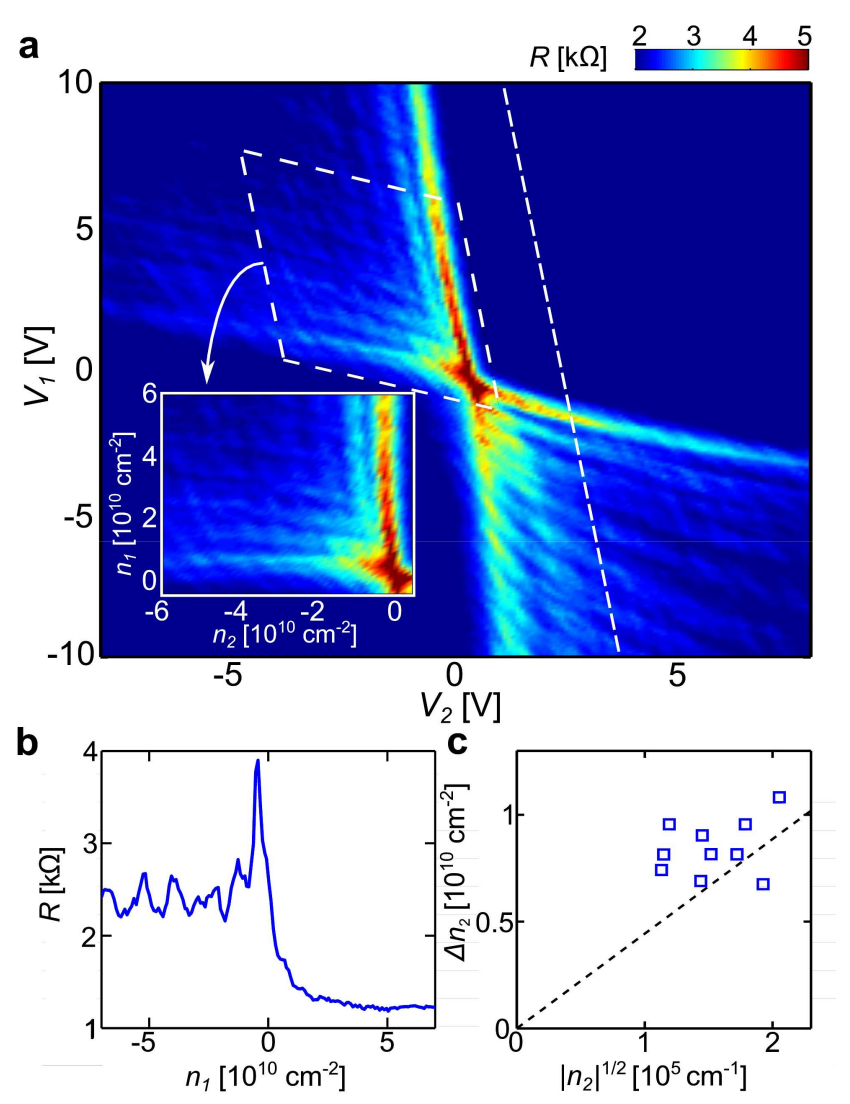

FIG. 2. (Color online) (a) Resistance oscillation measured at $T=0.25 \mathrm{~K}$ as a function of $V_{1}$ and $V_{2}$. Along the dashed white line, $n_{1}$ changes while $n_{2}$ is kept fixed at $2.82 \times 10^{10} \mathrm{~cm}^{-2}$. The data shown in a panel (b), and in Figs. 3 and 4 are taken along this line. The inset shows the plot of $R$ as a function of $n_{1}$ and $n_{2}$, for the $V_{1}$ and $V_{2}$ region corresponding to the parallelogram delimited by the dashed line. (b) $R\left(n_{1}\right)$ at fixed, positive $n_{2}$ shows oscillations for negative $n_{1}$, i.e., when a pn junction is present. (c) Oscillation period $\Delta n_{2}$, plotted as a function of $\sqrt{\left|n_{2}\right|}$. Empty squares show data taken at different values of $n_{1}$; the broken line represents the values of $\Delta n_{2}=2 \sqrt{\pi\left|n_{2}\right|} / L_{2}$ estimated form a simple particle-in-a-box approximation $\left(L_{2} \approx 800 \mathrm{~nm}\right)$.

bottom gate that overlaps with about half of the suspended length (see Fig. 1(e)). By screening the potential generated by a voltage applied to the conducting Si substrate, the bottom gate defines two regions (1 and 2, see Fig. 1(f)), whose carrier density and type can be controlled by applying voltages to the doped silicon substrate $\left(V_{1}\right)$ and to the local gate itself $\left(V_{2}\right)$. Transport measurements as a function of $V_{1}$ and $V_{2}$ were performed in a Heliox $\mathrm{He}^{3}$ system to characterize the device at different magnetic field $(B)$, bias $\left(V_{D C}\right)$, and temperature $(T)$. Prior to the measurements, the device was annealed at $4.2 \mathrm{~K}$ by passing 
a sufficiently large current through the graphene flake.

Fig. 2(a) shows the resistance $R$ measured at $T=0.25 \mathrm{~K}$ as a function of $V_{1}$ and $V_{2}$. Four quadrants can be identified, roughly corresponding to $V_{1}$ and $V_{2}$ having the same or opposite sign. When the sign is the same, no pn junction is present in the device: only either electrons or holes are accumulated in regions 1 and 2. A pn junction is present between region 1 and 2 when $V_{1}$ and $V_{2}$ have opposite sign. The borders of the different quadrants are not parallel to the $V_{1}$ and $V_{2}$ axis, because of the cross-talk between two gates: $V_{1}$ does not only change the density in region $1\left(n_{1}\right)$ but also - to a lesser extent- the density in region $2\left(n_{2}\right)$; similarly, $V_{2}$ also influences the density $n_{1}$. Although, in general, that the density is not spatially uniform in regions 1 and 2 (this is obvious when a $p n$ junction is present, in which case the carrier density vanishes at the interface between the two regions), accounting as much as possible for the effect of the cross-talk is useful to analyze the data. This can be done by looking at the gate and magnetic field dependence of the quantized Hall conductance plateaus in the unipolar regime, where $n_{1} \simeq n_{2}$ (i.e., when the density non uniformity is less pronounced). We find $n_{1}\left[10^{10} \mathrm{~cm}^{-2}\right]=1.0 \times V_{1}[\mathrm{~V}]+0.35 \times V_{2}[\mathrm{~V}]+0.5$ and $n_{2}\left[10^{10} \mathrm{~cm}^{-2}\right]=0.2 \times V_{1}$ $[\mathrm{V}]+1.4 \times V_{2}[\mathrm{~V}]-0.4$ (the constants account for the shift of charge neutrality point from $V_{1 / 2}=0 \mathrm{~V}$; the proportionality terms between $n_{1 / 2}$ and $V_{1 / 2}$ are in good agreement with the estimated geometrical capacitances). The resistance as a function of $n_{1}$ and $n_{2}$ defined in this way is shown in the inset of Fig. 2(a).

When $V_{1}$ and $V_{2}$ are biased with opposite polarity to create a pn junction, the resistance doubles as compared to when no pn junction is present (compare, e.g., the resistance for $n_{1}<0$ and $n_{1}>0$ in Fig. 2(b)). This shows that the $p n$ junction gives a large contribution to the total device resistance, despite the sizable device length (1.8 $\mu \mathrm{m}$ in total). In particular, the $p n$ junction contribution significantly larger as compared to previously studied $p n$ and pnp junctions on $\mathrm{SiO}_{2}$ substrates. ${ }^{20-23}$

Fig. 2(a) further shows that the resistance also oscillates as a function of $V_{1}$ and $V_{2}$ when a $p n$ junction is formed, in a way resembling the behavior of graphene pnp junctions on a $\mathrm{Si} / \mathrm{SiO}_{2}$ substrate. ${ }^{19,24}$ In that case, the oscillations were shown to originate from FabryPerot interference of Dirac electrons moving ballistically within the $(\approx 100 \mathrm{~nm}$ long) cavity defined by the pnp region. ${ }^{19,24,25}$ In our device, Fabry-Perot oscillations occur in cavities formed by the $p n$ junction and each of the two interfaces with the metal contacts, where carriers are also backscattered. ${ }^{26,27}$ Our device therefore consists of two Fabry-Perot cavities 


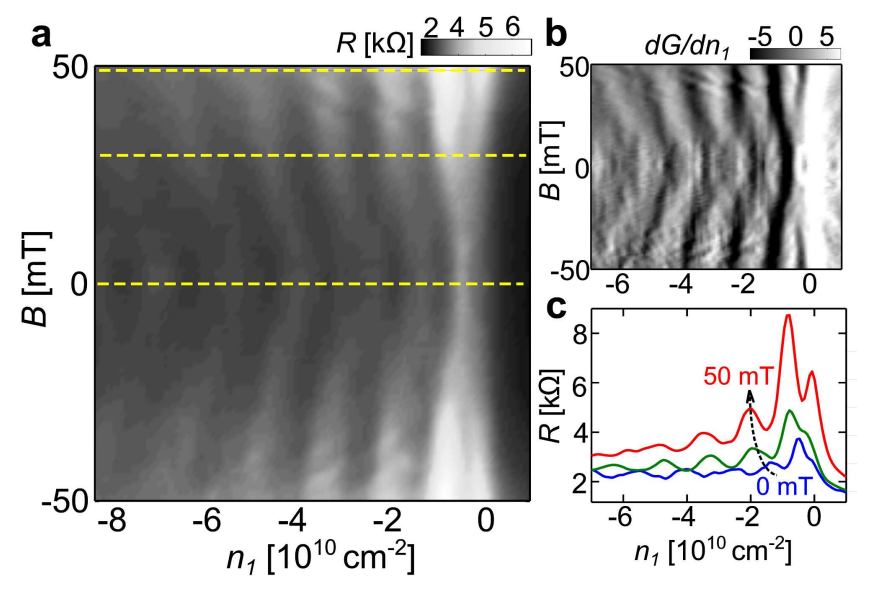

FIG. 3. (Color online) Magnetic field dependence of $R\left(n_{1}\right)$ measured at $T=0.25 \mathrm{~K}$, at fixed $n_{2}$, along the dashed line shown in Fig. 2(a). (a) Plot of $R\left(n_{1}, B\right)$, showing characteristic $\pi$-shift at $B=B^{*} \approx 20-30 \mathrm{mT}$. (b) Plot of $d G / d n_{1}$, as a function of $n_{1}$ and $B$, shown for comparison with similar data reported in the literature. In (a), the broken lines indicate the values of $B(B=0$, $30,50 \mathrm{mT}$ ) at which the data in (c) are measured.

connected in series, and the "checkerboard" pattern visible in Fig. 2(a) is a manifestation of interference in both cavities. The clear visibility of the oscillations directly in the resistance (Figs. 2(a) and 2(b)), without the need of derivating the data, is indicative of the high quality of the suspended $p n$ junction. ${ }^{19,24}$

An estimate of the oscillation period $\Delta n$ (i.e., the distance in density between two nearest resistance peaks or dips) is obtained by imposing that the dynamical phase acquired by an electron wave propagating back and forth in the cavity is equal to $2 \pi$, i.e. $\Delta\left(2 k_{F, i} L_{i}\right)=2 \pi$ (the subscript $i=1,2$ label the region). As $k_{F, i}=\sqrt{\pi n_{i}}$, we obtain $\Delta n_{i}=2 \sqrt{\pi n_{i}} / L_{i}$ (note that several previous references ${ }^{19,24,28}$ reported an incorrect expression, $\Delta n_{i}=4 \sqrt{\pi n_{i}} / L_{i}$, differing by a factor of 2 from ours). The dotted line in Fig. 2(c) represents the values of $\Delta n_{2}$ estimated using this formula for region $2\left(L_{2}=800 \mathrm{~nm}\right)$, and the open squares are the experimental values extracted from the most pronounced oscillations measured upon changing $n_{2}$ (a similar result is obtained for region 1). The order of magnitude and the trend in the data are well captured by the simple theoretical expression. The experimental values, however, are somewhat larger than expected, because the carrier density in the region close to the $p n$ junction is lower than the calculated value $n_{2}$. The lower density causes a smaller value of $k_{F}$, and therefore a smaller phase shift and an additional increase in carrier 

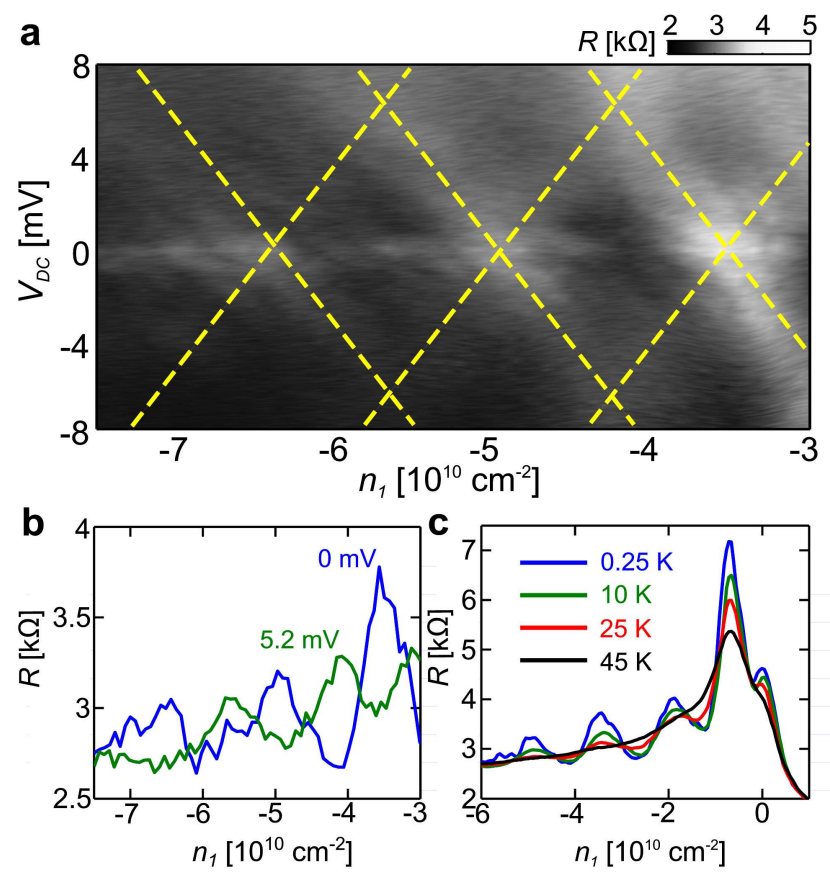

FIG. 4. (Color online) Energy dependence of the Fabry-Perot interference. (a) Resistance $R$ measured at $T=0.25 \mathrm{~K}$ as a function of bias $V_{D C}$ and $n_{1}$ (at fixed $n_{2}$ along a dashed line in Fig. 2(a)); the yellow dashed lines are guides to the eye . (b) Two representative curves of $R\left(n_{1}\right)$ measured at at $V_{D C}=0$ and $5.2 \mathrm{mV}$, showing a $\pi$ shift in the oscillation phase. (c) $T$-dependence of $R\left(n_{1}\right)$ measured at $V_{D C}=0 \mathrm{mV}$. All data in this figure were taken at $B=40 \mathrm{mT}$

density is needed to compensate for this effect.

The evolution of the oscillation phase upon increasing magnetic field $B$ (see Fig. 3) provides further evidence for the Fabry-Perot nature of the interference. ${ }^{19,24,25}$ Fig. 3(a) shows the $B$-dependence of the oscillations upon changing $n_{1}$ at fixed $n_{2}=2.82 \times 10^{10} \mathrm{~cm}^{-2}$ (i.e., by changing $V_{1}$ and $V_{2}$ along the dashed line depicted in Fig. 2(a)), which exhibits a $\pi$ phase shift at $B \equiv B^{*} \approx 20-30 \mathrm{mT}$ (varying $n_{2}$ at fixed $n_{1}$ gives comparable results). Fig. $3(\mathrm{~b})$ shows the same effect in the derivative of the conductance $(G=1 / R)$ with respect to $n_{1}$, and panel (c) illustrates the occurrence of the phase shift, with three individual slices of the color plot shown in (a), taken at $B=0,30$, and $50 \mathrm{mT}$.

As discussed for $p n p$ junctions, ${ }^{19,24,25}$ the phase shift originates from the unique properties of Dirac electrons, namely the angular dependence of the reflection probability at a pn junction, ${ }^{29,30}$ and the accumulation of a $\pi$ Berry phase along momentum-space trajectories that enclose the origin. ${ }^{31,32}$ For a given position of the Fermi energy, the electrons 
contributing predominantly to the Fabry-Perot resistance oscillations are those incident on the $p n$ junction with a certain transverse momentum $\left(k_{y 0}\right.$; the specific value depends on the density profile across the junction). ${ }^{24,25}$ Upon increasing the perpendicular magnetic field, the electron trajectories in the Fabry-Perot cavity are bent, and -in momentum spacethey eventually enclose the origin. ${ }^{24,25}$ When this happens, an additional Berry phase $\pi$ is acquired, causing the phase shift in the resistance oscillations. For $\simeq 100 \mathrm{~nm}$ long pnp junctions on substrate, the shift was found to occur at $B^{*} \approx 2 \hbar k_{y 0} / e L \approx 250-500 \mathrm{mT}^{19,24}$ Assuming a comparable value of $k_{y 0}$ (within a factor of 2-3), this is consistent with our observations: the phase shift occurs at an order of magnitude smaller $B^{*} \simeq 20-30 \mathrm{mT}$, corresponding to an order of magnitude longer cavity.

Finally, we discuss the characteristic energy scale of the resistance oscillations. Fig. 4(a) shows the differential resistance measured as a function of bias $V_{D C}$ and density $n_{1}$. Systematically, the position of the resistance peaks shifts linearly upon increasing $V_{D C}$, as expected for Fabry-Perot interference. ${ }^{26,27}$ The shift is also illustrated by Fig. 4(b), which compares measurements taken at $V_{D C}=0$ and $5.2 \mathrm{mV}$. From both Figs. 4(a) and 4(b), the bias needed to shift a maximum of differential resistance into a minimum is approximately $5 \mathrm{meV}$. We have also looked at the energy dependence of the oscillation by changing temperature, and found that the oscillations are washed out at about $40 \mathrm{~K}(\approx 3.5 \mathrm{meV})$. Since, owing to the non-uniform charge density, the level spacing in the cavity is somewhat larger than the particle-in-a-box value $h v_{F} / 2 L \approx 2.5 \mathrm{meV}$ (with $L \simeq 1 \mu \mathrm{m}$ and $v_{F}=10^{6} \mathrm{~m} / \mathrm{s}$ ), the energy scale found in the experiments is consistent with the simplest theoretical estimate.

We conclude that the behavior of our device is consistent with the presence of a $p n$ junction, and with transport occurring in the ballistic regime over a length comparable to the device size $(1.8 \mu \mathrm{m})$. The measurements therefore confirm that the fabrication technique that enables the realization of suspended graphene devices with local bottom gates preserves the high quality of the material. In the future, this technology will be applied to realize new graphene devices relying on the local control of the electrostatic potential and electric field, such as the nano-structures needed for the study of Veselago lensing, ${ }^{33}$ of collimation of electrons, ${ }^{34}$ and of topological confinement. ${ }^{15}$

We gratefully acknowledge A. Ferreira for technical support and financial support from the SNF, NCCR MaNEP, and NCCR QSIT. 


\section{REFERENCES}

${ }^{1}$ K. Bolotin, K. J. Sikes, Z. Jiang, M. Klima, G. Fudenberg, J. Hone, P. Kim, and H. L. Stormer, Solid State Communications 146, 351 (2008).

${ }^{2}$ X. Du, I. Skachko, B. A., and E. Y. Andrei, Nature Nanotechnology 3, 491 (2008).

${ }^{3}$ C. R. Dean, A. F. Young, I. Meric, C. Lee, L. Wang, S. Sorgenfrei, K. Watanabe, T. Taniguchi, P. Kim, K. L. Shepard, and J. Hone, Nature Nanotechnology 5 (2010).

${ }^{4}$ C. Dean, A. Young, L. Wang, I. Meric, G.-H. Lee, K. Watanabe, T. Taniguchi, K. Shepard, P. Kim, and J. Hone, Solid State Communications 152, 1275 (2012).

${ }^{5}$ R. T. Weitz, M. T. Allen, B. E. Feldman, J. Martin, and A. Yacoby, Science 330, 812 (2010).

${ }^{6}$ J. Velasco, L. Jing, W. Bao, Y. Lee, P. Kratz, V. Aji, M. Bockrath, C. N. Lau, C. Varma, R. Stillwell, D. Smirnov, F. Zhang, J. Jung, and A. H. MacDonald, Nature Nanotechnology 7, 156 (2012).

${ }^{7}$ M. T. Allen, J. Martin, and A. Yacoby, Nature Communications 3, 934 (2012).

${ }^{8}$ X. Du, I. Skachko, F. Duerr, A. Luican, and E. Y. Andrei, Nature 462, 192 (2009).

${ }^{9}$ K. I. Bolotin, F. Ghahari, M. D. Shulman, H. L. Stormer, and P. Kim, Nature 462, 196 (2009).

${ }^{10}$ C. R. Dean, A. F. Young, P. Cadden-Zimansky, L. Wang, H. Ren, K. Watanabe, T. Taniguchi, P. Kim, J. Hone, and K. L. Shepard, Nature Physics 7, 693 (2011).

${ }^{11}$ D.-K. Ki, V. I. Fal'ko, and A. F. Morpurgo, Submitted (2013).

${ }^{12}$ A. S. Mayorov, R. V. Gorbachev, S. V. Morozov, L. Britnell, R. Jalil, L. A. Ponomarenko, P. Blake, K. S. Novoselov, K. Watanabe, T. Taniguchi, and A. K. Geim, Nano Letters 11, 2396 (2011).

${ }^{13}$ L. Campos, A. Young, K. Surakitbovorn, K. Watanabe, T. Taniguchi, and P. JarilloHerrero, Nature Communications 3 (2012).

${ }^{14}$ D.-K. Ki and A. F. Morpurgo, In preparation (2013).

${ }^{15}$ I. Martin, Y. M. Blanter, and A. F. Morpurgo, Physical Review Letters 100, 036804 (2008).

${ }^{16}$ N. Tombros, A. Veligura, J. Junesch, J. J. van den Berg, P. J. Zomer, M. Wojtaszek, I. J. Vera-Marun, H. T. Jonkman, and B. J. van Wees, Journal of Applied Physics 109 (2011).

${ }^{17}$ N. Tombros, A. Veligura, J. Junesch, M. H. D. Guimares, I. J. Vera-Marun, H. T. Jonkman, 
and B. J. van Wees, Nature Physics 7, 697 (2011).

${ }^{18}$ D.-K. Ki and A. F. Morpurgo, Physical Review Letters 108, 266601 (2012).

${ }^{19}$ S.-G. Nam, D.-K. Ki, J. W. Park, Y. Kim, J. S. Kim, and H.-J. Lee, Nanotechnology 22, 415203 (2011).

${ }^{20}$ B. Huard, J. Sulpizio, N. Stander, K. Todd, B. Yang, and D. Goldhaber-Gordon, Physical Review Letters 98, 8 (2007).

${ }^{21}$ J. R. Williams, L. Dicarlo, and C. M. Marcus, Science 317, 638 (2007).

${ }^{22}$ R. V. Gorbachev, A. S. Mayorov, A. K. Savchenko, D. W. Horsell, and F. Guinea, Nano letters 8, 1995 (2008).

${ }^{23}$ N. Stander, B. Huard, and D. Goldhaber-Gordon, Physical Review Letters 102, 1 (2009).

${ }^{24}$ A. F. Young and P. Kim, Nature Physics 5, 222 (2009).

${ }^{25}$ A. Shytov, M. Rudner, and L. Levitov, Physical Review Letters 101, 10 (2008).

${ }^{26}$ F. Miao, S. Wijeratne, Y. Zhang, U. C. Coskun, W. Bao, and C. N. Lau, Science 317, 1530 (2007).

${ }^{27}$ S. Cho and M. Fuhrer, Nano Research 4, 385 (2011).

${ }^{28}$ J. Velasco, G. Liu, W. Bao, and C. Ning Lau, New Journal of Physics 11, 095008 (2009).

${ }^{29}$ M. I. Katsnelson, K. S. Novoselov, and A. K. Geim, Nature Physics 2, 620 (2006).

${ }^{30}$ V. Cheianov and V. Fal'ko, Physical Review B 74, 1 (2006).

${ }^{31}$ K. S. Novoselov, A. K. Geim, S. V. Morozov, D. Jiang, M. I. Katsnelson, I. V. Grigorieva, S. V. Dubonos, and A. A. Firsov, Nature 438, 197 (2005).

${ }^{32}$ Y. Zhang, Y.-W. Tan, H. L. Stormer, and P. Kim, Nature 438, 201 (2005).

${ }^{33}$ V. V. Cheianov, V. Fal'ko, and B. L. Altshuler, Science 315, 1252 (2007).

${ }^{34}$ C.-H. Park, Y.-W. Son, L. Yang, M. L. Cohen, and S. G. Louie, Nano Letters 8, 2920 (2008). 